\title{
ATEROESCLEROSIS ASOCIADA A INFECCION POR Chlamydophila pneumoniae: INTERACCIÓN ENTRE EL SER HUMANO Y UNA BACTERIA
}

\section{ATHEROSCLEROSIS ASSOCIATED TO INFECTION BY Chlamydophila pneumoniae: INTERACTION BETWEEN HUMANS AND BACTERIA}

\author{
TITULO CORTO: ATEROESCLEROSIS ASOCIADA A INFECCIÓN POR \\ Chlamydophila pneumoniae
}

Alvaro Gudiño-Gomezjurado ${ }^{1}$ y Marco Gudiño-Gomezjurado ${ }^{2}$

Recibido en Octubre 15 de 2014

Aceptado en Diciembre 03 de 2014

\section{RESUMEN}

La ateroesclerosis y sus complicaciones constituyen una de las mayores causas de morbi-mortalidad a nivel mundial. Sin embargo, solo la mitad de los casos se justifica por los factores de riesgo tradicionales. No obstante, desde hace casi cinco décadas se ha intentado encontrar causas no tradicionales asociadas a la enfermedad ateroesclerótica. Varios agentes infecciosos han emergido como posibles candidatos centralizándose la investigación en Chlamydophila pneumoniae por su capacidad de infectar las células endoteliales durante el proceso ateroesclerótico. A lo largo del tiempo, múltiples estudios han tratado de probar la causalidad de $C$. pneumoniae en el proceso ateroesclerótico y sus complicaciones. A pesar de esto, hasta la fecha las conclusiones son ambiguas y de poca relevancia para la práctica clínica diaria.

Palabras clave: Aterosclerosis; Chlamydophila pneumoniae; Enfermedades Cardiovasculares (fuente: DeCS)

\section{ABSTRACT}

Atherosclerosis and its complications are a major cause of morbidity and mortality worldwide. However, only half of the cases are justified by traditional risk factors. Nevertheless for almost five decades ago, has tried to find non-traditional causes associated with atherosclerotic disease. Various infectious agents have emerged as potential candidates being centralized research Chlamydophila pneumoniae by its ability to infect endothelial cells in the atherosclerotic process. Over time many studies have tried to prove the causality of $C$. pneumoniae in the atherosclerotic process and its complications. Despite this, to date the findings are ambiguous and of little relevance to daily clinical practice.

Keywords: Atherosclerosis; Chlamydophila pneumoniae; Cardiovascular Diseases (fuente: DeCS) 


\section{INTRODUCCIÓN}

Se define a la ateroesclerosis a un proceso en el cual se $\mathcal{S p r o d u c e ~ e l ~ d e p o ́ s i t o ~ y ~ p o s t e r i o r ~ i n f i l t r a c i o ́ n ~ d e ~ l i ́ p i d o s ~}$ en la íntima de las arterias de mediano y gran calibre. Actualmente, las complicaciones secundarias al proceso ateroesclerótico, constituyen un verdadero problema para la salud pública tanto en países desarrollados y en los países en vías de desarrollo. Según datos de la Organización Mundial de la Salud, 16.7 millones de muertes al año se producen por enfermedades cardiovasculares a nivel mundial, y en Colombia, según datos del Instituto Nacional de Salud, entre los años 1998-2011 se registraron 628.630 muertes por enfermedad cardiovascular, representando al $23 \%$ del total de muertes registradas en ese país ${ }^{1-3}$.

Frente a estos antecedentes, solo entre el $50 \%$ y el $70 \%$ de los casos se deben a los factores de riesgo tradicionales: Diabetes Mellitus tipo 2 (DM tipo 2), hipertensión arterial (HTA), consumo de cigarrillo, la edad, el sexo, hiperhomocisteinemia y los antecedentes familiares ${ }^{4-7}$.

Desde 1968 diferentes estudios se han llevado a cabo con el fin de determinar otros factores de riesgo asociados al desarrollo de la enfermedad ateroesclerótica; con lo que fue posible identificar a varios virus cardiotropos, como el Coxsaquie B4, herpes virus, citomegalovirus, virus de la Hepatitis A o bacterias como Micoplasma pneumoniae, Bacteroides gingivalis o Helicobacter pylori ${ }^{1}$.

Siguiendo esta línea, uno de los principales agentes que ha llamado la atención por su capacidad aterogénica es Chlamydophila pneumoniae, ya que tiene la capacidad de infectar las células endoteliales y los macrófagos circulantes y desencadenar un proceso inflamatorio crónico a nivel endotelial ${ }^{8-10}$.

Con base en estos antecedentes, esta revisión tiene por objetivo actualizar la información referente al papel que tiene Chlamydophila pneumoniae dentro del proceso ateroesclerótico y evidenciar si el tratamiento antibiótico podría ser una nueva estrategia terapéutica para el manejo de las enfermedades cardiovasculares.

Con estos antecedentes se realizó este artículo de revisión con el objetivo de determinar, con base en la evidencia actual, si la infección por $C$ pneumoniae es factor de riesgo para el desarrollo de ateroesclerosis y cuál es su relevancia clínica sobre las enfermedades cardiovasculares. Para este fin se efectuó la búsqueda de artículos originales y de revisión publicados en inglés a partir del año 1998 hasta el año 2013 en la base de datos MEDLINE y empleando el buscador PUBMED. Los descriptores de búsqueda fueron "Chlamydophila pneumoniae" and "cardiovascular disease".

\section{RESULTADOS}

La ateroesclerosis es un proceso inflamatorio crónico caracterizado por el depósito de material lipídico a nivel de la íntima de las arterias de mediano y gran calibre. Una vez que se produce el depósito de las partículas de colesterol LDL en el endotelio arterial, se desencadena el proceso inflamatorio, lo que atrae a los monocitos circulantes, los cuales penetran en el endotelio arterial, donde se transforman en macrófagos, los cuales fagocitan al colesterol LDL y se convierten en células espumosas. Estas células espumosas, conjuntamente con el núcleo lipídico y la capa fibromuscular constituyen la placa ateromatosa, la cual por varios factores como la hiperlipidemia, el tabaquismo, el antecedente de Hipertensión arterial o Diabetes Mellitus tipo 2, la homocisteinemia o las infecciones por $C$ pneumoniae, pueden perpetuar el proceso inflamatorio provocando la inestabilización de la placa ateroesclerótica lo que clínicamente se traduce en la presentación eventos cardiovasculares ${ }^{1,6,7,9}$.

\section{Chlamydophila pneumoniae}

Es una bacteria perteneciente al filo Chlamydiae, de la familia Chamydiaceae, relacionado, principalmente, con infecciones del tracto respiratorio superior en el ser humano ${ }^{11}$. Su ciclo de vida es bifásico, ya que una vez que ingresa al huésped en forma de corpúsculo elemental (CE), metabólicamente inactivo, debe desarrollarse a corpúsculo reticular (CR) que es la forma intracelular y metabólicamente activa ${ }^{6,12,13}$.

Una vez que el CE ingresa al interior de la célula hospedera rápidamente se forma el $\mathrm{CR}$ debido a divisiones sucesivas por fisión binaria en un período de $48 \mathrm{~h}$ a $72 \mathrm{~h}$, lo que origina nuevos CR que infectarán a otras células ${ }^{6,12}$. Sin embargo, cuando las condiciones para el desarrollo bacteriano no son adecuadas se produce una forma aberrante del CR denominada forma intermedia, la cual no es infecciosa pero permanece viable dentro de la célula, por un mecanismo ligado a una mayor concentración de interferón gamma (IFN- $\gamma)^{13}$. Estas formas intermedias son las que están ligadas directamente con el proceso ateroesclerótico. 


\section{Patogénesis de la ateroesclerosis y su relación con C. pneumoniae}

La infección por $C$. pneumoniae inicia con la colonización del tracto respiratorio a través de las células del epitelio columnar o de transición que tapiza el árbol respiratorio ${ }^{14}$. Esta bacteria por su capacidad de evadir la respuesta inmune del huésped se disemina desde los pulmones hacia la sangre por intermedio de las células mononucleares. Por intermedio de estas células, la bacteria puede localizarse en varios tejidos como las arterias y comprometer al endotelio vascular y a las células musculares lisas ${ }^{6,14,15}$.

Una vez que $C$. pneumoniae infecta las células endoteliales el hospedador produce una respuesta inmunológica frente al antígeno de superficie OMP2 de la bacteria con la consecuente liberación de múltiples mediadores proinflamatorios por parte del hospedador como las interleucinas (IL) IL-1, IL-6, IL-8, IL-12, IL-17; Factor de necrosis tumoral- alfa (TNF- $\alpha$ ), la proteína quimiotáctica de los monocitos tipo 1 (MCP-1) e INF- $\gamma$. También incrementa la concentración de moléculas de adhesión como: la molécula de adhesión leucocitaria tipo 1 (ELAM1), molécula de adhesión intercelular tipo 1 (ICAM-1) y la molécula de adhesión de las células vasculares tipo 1 (VCAM-1) que permiten la migración de leucocitos y monocitos a través del endotelio vascular además, de varios de factores de crecimiento como el factor de crecimiento derivado de las plaquetas (PDGF), el factor nuclear- $\kappa \beta$ (NF-к $\beta$ ) que inducen el desarrollo de las células musculares lisas y producción de colágeno, fibrina y proteoglicanos por activación de las vías de la proteína cinasa activada por mitógenos ERK1/2MAPK o p44/p42 MAPK y de los receptores toll like receptors tipo 2 y tipo 4 ( TLR-2) y (TLR-4) $)^{1,12,16-20}$.

Por otro lado, Kalayoglu y Byrne ${ }^{21}$, demostraron que por acción del LPS bacteriano los macrófagos bacterianos engloban más partículas de colesterol LDL e induce a la oxidación de este, lo que da origen a las células espumosas que forman parte del núcleo de la placa ateroesclerótica. Cuando se acumula suficiente cantidad de lípidos el núcleo de la placa se hace necrótico y posteriormente se calcifica incrementando aún más la liberación de citocinas y de mediadores inflamatorios ${ }^{12,16}$.

Además, la proteína de choque de calor cHsp 60 interviene en el proceso de desestabilización de la placa ateroesclerótica debido a la hiperestimulación de los macrófagos y por el mimetismo que tiene con la proteína de origen humana, lo que hace que aumenten los niveles de TNF- $\alpha$ y de las metaloproteinasas de la matriz (MMP), lo que a la larga conlleva a una mayor producción del anión superóxido provocando la disminución de óxido nítrico y la consecuente descomposición del colágeno y la rotura de la placa ${ }^{6,10,12,16}$.

Sin embargo, no solamente la inmunidad celular interviene dentro del proceso aterogénico inducido por C. pneumonie, sino que también por intermedio de esta bacteria, se produce activación de la inmunidad humoral que desencadena producción cruzada de anticuerpos expresados sobre los fibroblastos de la placa ateromatosa $\mathrm{a}^{22,23}$.

\section{Estudios seroepidemiológicos}

A lo largo de los años, múltiples estudios realizados por diferentes métodos diagnósticos como inmunohistoquímica, microscopia electrónica o pruebas serológicas han identificado de forma significativa la presencia de $C$. pneumoniae en las placas de pacientes que sufren enfermedad ateroesclerótica comparado con testigos sanos que no presentan esta enfermedad ${ }^{10,12}$.

En 1988 se identificó por primera vez que los pacientes con enfermedad coronaria establecida y con antecedente de Infarto Agudo de Miocardio (IAM) los niveles de anticuerpos anti IgA para C. pneumoniae fueron significativamente más altos en relación a los controles sanos ${ }^{10,12}$.

A partir de este primer hallazgo se diseñaron múltiples estudios retrospectivos los cuales evidenciaron que los niveles elevados de anticuerpos anti $C$. pneumoniae en pacientes con enfermedad ateroesclerótica establecida tenían una asociación entre 1.6 a 7.4 veces por encima de los controles sanos ${ }^{3,4}$. Sin embargo, en el año 2003 se realizó un metaanalisis de 29 estudios prospectivos en el cual no se demostró ninguna asociación ${ }^{12,24}$.

No obstante, otro metaanalisis publicado en el año 2013 por Chen et al. ${ }^{25}$ demostraron que los niveles elevados de IgA (OR, 2.33; $95 \%$ IC: 1.76 to 3.08 ) e IgG (OR, 1.61; $95 \%$ IC: 1.34 to 1.94), anti C. pneumoniae están significativamente asociados con el riesgo de desarrollar enfermedad cerebrovascular.

Mediante la Reacción en Cadena de la Polimerasa (PCR) se detectó la presencia de $C$. pneumoniae en los monocitos de sangre periférica de personas infectadas y que tenían enfermedad coronaria establecida ${ }^{26}$. La prevalencia encontrada en los diferentes estudios fue del $59 \%$ en los casos con una variabilidad entre el $4 \%$ y el

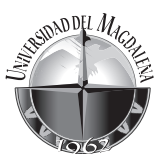


$87 \%$ comparado con el $46 \%$ de los controles con una variabilidad entre el $0 \%$ y el $50 \%$ siendo la sensibilidad y la especificidad de esta técnica del $76.5 \%$ y del $99 \%$, respectivamente ${ }^{12,27}$

\section{Estudios histopatológicos}

En el año 1992 se describió por primera vez la presencia de C. pneumoniae en arterias ateromatosas mediante microscopia electrónica e inmunohistoquímica ${ }^{12}$.

Se cree que la presencia de $C$. pneumoniae está directamente relacionada con el desarrollo e inestabilización de la placa ateroesclerótica en varios lechos arteriales como las arterias iliacas, las carótidas o la cerebral media. Así como también con otros procesos degenerativos vasculares como los aneurismas aórticos. ${ }^{5,27-30}$.

Una revisión sistemática de 43 estudios histopatológicos ${ }^{12}$, procesados por diferentes métodos diagnósticos como son: cultivos de tejidos, microscopia electrónica, inmunofluoresencia, inmunocitoquímica o hibridación in situ permitieron evidenciar la presencia de $C$. pneumoniae en el $46 \%$ de los casos, comparado con menos del $1 \%$ de los controles. No obstante, estos hallazgos poseen una gran variabilidad de resultados ya que los datos positivos oscilan entre el $2 \%$ y el $100 \%$ de los casos ${ }^{4,12}$.

Una de las mayores desventajas de este método de detección es la pobre estandarización y el alto grado de subjetividad ya que, es un procedimiento altamente dependiente del observador que lo realiza. Por otro lado, no permite identificar fehacientemente que $C$. pneumoniae tenga un papel activo en el proceso ateroesclerótico ya que simplemente su hallazgo en las placas ateroescleróticas pueden ser la de un "huésped inocente"12,24,31.

\section{Estudios en animales}

Los primeros experimentos en animales se llevaron a cabo en Nueva Zelanda en el año de 1998 donde un grupo de conejos sanos se sometió a una dieta aterogénica y a otro grupo a la misma dieta aterogénica mas la inoculación intranasal de C. pneumoniae. El grupo de conejos tratados solamente con dieta enriquecida en lípidos no desarrollo ateroesclerosis mientras que el grupo inoculado con $C$. pneumoniae desarrolló rápidamente placas ateroescleróticas a nivel aórtico ${ }^{9,12,24}$.

Pese a estos hallazgos, otros estudios como el realizado en el año 2001 por Aalto-Setälä K et al., ${ }^{32}$, demostraron que la presencia de $C$. pneumoniae no aceleró los cambio aterogénicos en la raíz aórtica en ratones con deficiencia de ApoE aunque si evidenciaron que $C$. pneumoniae inducía un proceso inflamatorio crónico, especialmente en aquellos modelos sometidos a dieta enriquecida con colesterol.

\section{Intervenciones farmacológicas}

Los primeros datos sobre la eficacia del tratamiento frente a $C$. pneumoniae para la prevención de eventos cardiovasculares provinieron de estudios de sensibilidad in vitro y observacionales ${ }^{33}$. Uno de los primeros fue realizado por Muhlestein ${ }^{34}$ y Meier et al., ${ }^{35}$ quienes demostraron que los pacientes dosificados con tetraciclinas tuvieron menos riesgo de presentar un evento cardiovascular en comparación con los que no habían recibido estos antimicrobianos. Sin embargo, estudios con similar diseño, como el llevado a cabo por Jackson et al., ${ }^{36}$, Fong ${ }^{37}$ y Jackson et al. ${ }^{38}$ quienes no identificaron ninguna reducción de eventos cardiovasculares con el uso previo de eritromicina, tetraciclina o doxiciciclina. Una de las mayores dificultades respecto a estos estudios fue que los antimicrobianos fueron usados por diversas causas y no específicamente para el tratamiento de $C$. pneumoniae, lo que produjo una mayor heterogeneidad en los resultados 34,39 .

El primer estudio prospectivo en prevención secundaria fue realizado en el año de 1997 por Gupta et al., ${ }^{40}$ en el cual 60 pacientes con antecedente de IAM y con títulos de antiecuerpos > 64 para anti IgG de $C$. pneumoniae recibieron azitromicina o placebo. Después de 18 meses de seguimiento la incidencia de nuevos eventos cardiovasculares fue 5 veces menor en el grupo tratado con azitromicina respecto al grupo placebo. Sin embargo, este primer estudio piloto fue criticado debido a fallas metodológicas en la randomización de los pacientes en el grupo control ya que se incluyó pacientes que no presentaban títulos elevados de anticuerpos anti $C$. pneumoniae ${ }^{39,41}$.

Posteriormente, se diseñaron diversos estudios para determinar si el tratamiento para $C$. pneumoniae era efectivo en la prevención secundaria de nuevos eventos cardiovasculares con resultados variables. Así, el estudio realizado por Sinisalo et al., ${ }^{42}$ (Clarithromycin in Acute Coronary Syndrome patients in Finland) permitió evidenciar que el grupo tratado con claritromicina redujo el número de eventos cardiovasculares isquémicos frente al grupo placebo. Sin embargo, el estudio ANTIBIO (The Antibiotic Therapy After Acute Myocardial Infarction) en el cual se comparó el uso de roxitromicina y placebo en 
872 pacientes con antecedente de IAM tras 6 semanas de tratamiento no evidenció diferencia estadísticamente significativa en la disminución de la mortalidad por IAM (OR 1.1 IC 95\% 0,6- 1.9) ${ }^{34}$.

Finalmente, otros trabajos prospectivos a gran escala como son los estudios WIZARD (Weekly Intervention with Zithromax for atherosclerosis and its related disorders), AZACS (Azithromycin in Acute Coronary Sydrome), ROXIS (Roxithromycin in Ischemic Syndromes), CLARICOR y ACADEMIC (The Azithromycin in Coronary Artery Disease: Elimination of Myocardial Infection with Chlamydia) demostraron que ningún tratamiento específico para $C$. pneumoniae era efectivo para la prevención secundaria de nuevos eventos cardiovasculares o de reestenosis en pacientes sometidos a angioplastia con stent ${ }^{12,15,24,34,43-49}$. Varios estudios también evaluaron el tratamiento para C. pneumoniae en otras complicaciones derivadas de la enfermedad ateroesclerotica como es el uso de roxitromicina para evaluar si disminuye el engrosamiento de la íntima en la arteria carótida, o el uso de roxitromicina o doxiciclina como alternativas en el manejo de los aneurismas abdominales. En ambos casos los resultados no son concluyentes, evidenciándose escaso o nulo efecto del tratamiento antibiótico sobre estas variables ${ }^{50-52}$.

Frente a estos hallazgos es importante determinar que hasta la fecha no se conoce cuál sería el mejor esquema de tratamiento antimicrobiano y su tiempo ideal de duración ya que las pruebas de sensibilidad y eficacia para el tratamiento de $C$. pneumoniae provienen de datos obtenidos a partir de estudios in vitro ${ }^{24,53}$. Solamente el estudio STAMINA (South Thames Trial of Antibiotics in Myocardial Infarction and Unstable Angina) evaluó dos esquemas de tratamiento diferentes en el cual no se identificó ninguna diferencia aparente entre el uso de azitromicina o de amoxicilina ${ }^{34,54,55}$.

\section{DISCUSIÓN}

Con base en lo expuesto, se puede concluir que la relación entre la infección por $C$. pneumoniae y el desarrollo de enfermedades cardiovasculares es ambigua, de ahí que se hace necesario la realización de un mayor número de estudios a nivel genético y de expresión proteíca para determinar con mayor certeza la interacción entre este microorganismo y la enfermedad cardiovascular.

En relación con los estudios seroepidemiológicos, podemos aseverar que la evidencia es insuficiente para confirmar que la infección por $C$. pneumoniae es un factor de riesgo para el desarrollo de ateroesclerosis ya que existe una gran heterogeneidad entre los resultados de los diferentes estudios retrospectivos y una pobre correlación demostrada en los estudios prospectivos. Además, si bien la sensibilidad y la especificidad de los estudios por PCR son aceptables, la sola identificación de $C$. pneumoniae por este método en monocitos de sangre periférica no indica una relación directa de causalidad entre la infección por este organismo y el proceso ateroesclerótico.

En lo que respecta a los estudios histopatológicos no proveen evidencia suficiente para aseverar que $C$. pneumoniae per se, constituya un factor indispensable en la formación y posterior inestabilización de la placa ateroesclerótica por lo que si bien existe indicios de que $C$. pneumoniae tiene un rol como coadyuvante en mantener el proceso inflamatorio crónico a nivel endotelial, no existe evidencia suficiente que demuestre que este microorganismo por si solo sea capaz de iniciar el proceso ateroesclerótico.

Finalmente, en lo que respecta a las posibilidades terapéuticas y fundamentados en la evidencia expuesta, se puede decir que el uso de antibioticoterapia específica para $C$. pneumoniae no es efectiva para la prevención primaria y secundaria de eventos cardiovasculares.

\section{REFERENCIAS BIBLIOGRÁFICAS}

1. Quinn TC. Does Chlamydia pneumoniae cause coronary heart disease?. Curr Opin Infect Dis. 1998 Jun;11 (3):301-7.

2. García-Elorriaga G, Del Rey-Pineda G. Human immunodeficiency virus, atherosclerosis and Chlamydophila pneumoniae. World J Clin Infect Dis. 2012 August 25; 2(4): 54-62.

3. Colombia. Instituto Nacional de Salud. Enfermedad cardiovascular, principal causa de muerte en Colombia. Boletín $\mathrm{N}^{\circ}$ 1, (Diciembre 9 de 2013).

4. Dunne M. The evolving relationship between Chlamydia pneumoniae and atherosclerosis. Curr Opin Infect Dis. 2000 Dec;13(6):583-591.

5. Blasi F, Fagetti L, Allegra L. Chlamydia pneumoniae detection in atherosclerotic plaques in Italy. J Infect Dis. 2000 Jun;181 (Suppl 3):444-6.

6. Di Pietro M, Filardo S, De Santis F, Sessa R. Chlamydia pneumoniae infection in atherosclerotic lesion development through oxidative stress: a brief overview. Int J Mol Sci. 2013 Jul; 14(7):15105-20. doi: 10.3390/ijms140715105.

7. Wiesli P1, Maly FE, Meniconi A, Czerwenka W, Hoffmann $\mathrm{U}$, Vetter W, et al. Chlamydia pneumoniae seropositivity and hyperhomocysteinemia are linked in patients with atherosclerosis. Clin Chem. 2001;47(7):1304-6. 
8. Bayram A, Erdoğan MB, Ekși F, Yamak B. Demonstration of Chlamydophila pneumoniae, Mycoplasma pneumoniae, Cytomegalovirus, and Epstein-Barr virus in atherosclerotic coronary arteries, nonrheumatic calcific aortic and rheumatic stenotic mitral valves by polymerase chain reaction. Anadolu Kardiyol Derg. 2011 May;11(3):237-43. doi: 10.5152/akd.2011.057. Epub 2011 Apr 5.

9. Gagliardi RJ1, Silveira DR, Caffaro RA, Santos VP, CaiaffaFilho HH. Chlamydia pneumoniae and symptomatic carotid atherosclerotic plaque: a prospective study.Arq Neuropsiquiatr. 2007 Jun;65(2B):385-9.

10. Joshi R, Khandelwal B, Joshi D, Gupta OP. Chlamydophila pneumoniae infection and cardiovascular disease. N Am J Med Sci. 2013 Mar;5(3):169-81. doi: 10.4103/19472714.109178.

11. Dean D, Neumayr L, Kelly DM, Ballas SK, Kleman K, Robertson S, et al. Chlamydia pneumoniae and acute chest syndrome in patients with sickle cell disease.J Pediatr Hematol Oncol. 2003 Jan;25(1):46-55.

12. Watson C, Alp NJ. Role of Chlamydia pneumoniae in atherosclerosis. Clin Sci (Lond). 2008 Apr;114(8):509-31. doi: 10.1042/CS20070298.

13. Beatty WL, Belanger TA, Desai AA, Morrison RP, Byrne GI. Tryptophan depletion as a mechanism of gamma interferon-mediated chlamydial persistence. Infect Immun. 1994 Sep;62 (9):3705-11.

14. Kumar S, Hammerschlag MR. Acute respiratory infection due to Chlamydia pneumoniae: current status of diagnostic methods. Clin Infect Dis. 2007 Feb 15;44(4):568-76. Epub 2007 Jan 18.

15. Franco RR, Bodanese LC, Repetto G, Piccoli Jda C, Wiehe $\mathrm{M}$, Bonato $\mathrm{C}$, et al. Inflammatory markers and antichlamydial antibodies in patients with metabolic syndrome. Arq Bras Cardiol. 2011 Feb;96(2):134-9. Epub 2010 Nov 12.

16. Ljungh $\AA$. Atherosclerosis and chlamydophila (chlamydia) pneumoniae. From friends to foes 2005: 23.

17. Benagiano M, Munari F, Ciervo A, Amedei A, Paccani SR, Mancini F, et al. Chlamydophila pneumoniae phospholipase D (CpPLD) drives Th17 inflammation in human atherosclerosis. Proc Natl Acad Sci U S A. 2012 Jan 24;109(4):1222-7. doi: 10.1073/pnas.1111833109. Epub 2012 Jan 9.

18. Huang CY, Shih CM, Tsao NW, Chen YH, Li CY, Chang YJ, et al. GroEL1, from Chlamydia pneumoniae, induces vascular adhesion molecule 1 expression by p37(AUF1) in endothelial cells and hypercholesterolemic rabbit. PLoS One. 2012;7(8):e42808. doi: 10.1371/journal. pone.0042808. Epub 2012 Aug 10.
19. Chahine MN, Deniset J, Dibrov E, Hirono S, Blackwood DP, Austria JA, et al. Oxidized LDL promotes the mitogenic actions of Chlamydia pneumoniae in vascular smooth muscle cells. Cardiovasc Res. 2011 Dec 1;92(3):476-83. doi: 10.1093/cvr/cvr251.

20. Kreutmayer S, Csordas A, Kern J, Maass V, Almanzar G, Offterdinger $\mathrm{M}$, et al. Chlamydia pneumoniae infection acts as an endothelial stressor with the potential to initiate the earliest heatshock protein 60-dependent inflammatory stage of atherosclerosis. Cell Stress Chaperones. 2013 May;18(3):259-68. doi: 10.1007/ s12192-012-0378-7.

21. Kalayoglu M, Byrne G. Induction of macrophage foam cell formation by Chlamydia pneumoniae. J Infect Dis. 1998;(177):725-9

22. Canducci F, Saita D, Foglieni C, Piscopiello MR, Chiesa $\mathrm{R}$, Colombo A, et al. Cross-reacting antibacterial autoantibodies are produced within coronary atherosclerotic plaques of acutecoronary syndrome patients. PLoS One. 2012;7(8):e42283. doi: 10.1371/journal.pone.0042283. Epub 2012 Aug 6.

23. Nazmi A, Diez-Roux AV, Jenny NS, Tsai MY, Szklo $\mathrm{M}$, Aiello AE. The influence of persistent pathogens on circulating levels of inflammatory markers: a cross-sectional analysisfrom the Multi-Ethnic Study of Atherosclerosis. BMC Public Health. 2010 Nov;10:706. doi: 10.1186/1471-2458-10-706.

24. Honarmand H. Atherosclerosis Induced by Chlamydophila pneumoniae: A Controversial Theory.Interdiscip Perspect Infect Dis. 2013; 2013: 941392. doi: 10.1155/2013/941392. Epub 2013 Jul 17.

25. Chen J, Zhu M, Ma G, Zhao Z, Sun Z. Chlamydia pneumoniae infection and cerebrovascular disease: a systematic review and meta-analysis. BMC Neurol. 2013 Nov 21;13:183. doi: 10.1186/1471-2377-13-183.

26. Fallah F, Eslami G, Bootorabi M, Kazemi B, Goudarzi H, Mozaheri E. The isolation of chlamydia pneumoniae in atherosclerosis patients in Iran by PCR method. Gene Ther Mol Biol. 2000;10: 109-112.

27. Frishman WH, Ismail AA. Role of infection in atherosclerosis and coronary artery disease: a new therapeutic target?Cardiol Rev. 2002 Jul-Aug;10 (4):199-210.

28. Virok D, Kis Z, Karai L, Intzedy L, Burian K, Szabo A, et al. Chlamydia pneumoniae in atherosclerotic middle cerebral artery. Stroke. 2001 Sep;32 (9):1973-6.

29. Zeman K, Pospíšil L, Čanderle J, Štroblová H, Leypold J, Gregor, et al. Direct and indirect evidence of chlamydia pneumoniae in patients with significant stenosis of a. Carotis of atherosclerotic origin. Scripta medica (Brno). 2004 Jun; 77 (3): 173-180. 
30. Ramires JA, Higuchi Mde L. Mycoplasma pneumoniae and Chlamydia pneumoniae are associated to inflammation and rupture of the atherosclerotic coronary plaque. Rev Esp Cardiol. 2002;55 (S1):2-9.

31. Ieven MM, Hoymans VY. Involvement of Chlamydia pneumoniae in atherosclerosis: more evidence for lack of evidence. J Clin Microbiol. 2005 Jan;43 (1):19-24.

32. Aalto-Setälä, Laitinen K, Erkkilä L, Leinonen $\mathrm{M}$, Jauhiainen M, Ehnholm C. Chlamydia pneumoniae Does Not Increase Atherosclerosis in the Aortic Root of Apolipoprotein E-Deficient Mice. Vasc Biol Arterioscler 2001; 21:578-584.

33. Kutlin A, Roblin PM, Hammerschlag MR. In vitro activities of azithromycin and ofloxacin against Chlamydia pneumoniae in a continuous-infection model. Antimicrob Agents Chemother. 1999 Sep; 43 (9):2268-72.

34. Muhlestein JB. Antibiotic treatment of atherosclerosis. Curr Opin Lipidol. 2003 Dec; 14(6):605-14.

35. Meier CR, Derby LE, Jick SS, Vasilakis C, Jick H. Antibiotics and risk of subsequent first-time acute myocardial infarction. JAMA. 1999 Feb; 281 (5):427-31.

36. Jackson LA, Smith NL, Heckbert SR, Grayston JT, Siscovick DS, Psaty BM. Past use of erythromycin, tetracycline, or doxycycline is not associated with risk of first myocardial infarction. J Infect Dis. 2000 Jun;181 (S3):563-5.

37. Fong IW. Emerging relations between infectious diseases and coronary artery disease and atherosclerosis. CMAJ. 2000 Jul 11;163 (1):49-56.

38. Jackson LA, Smith NL, Heckbert SR, Grayston JT, Siscovick DS, Psaty BM. Lack of association between first myocardial infarction and past use of erythromycin, tetracycline, or doxycycline. Emerg Infect Dis. 1999 Apr;5 (2):281-4.

39. Ngeh J, Anand V, Gupta S. Chlamydia pneumoniae and atherosclerosis -- what we know and what we don't. Clin Microbiol Infect. 2002 Jan; 8(1):2-13.

40. Gupta S, Camm AJ. Chronic infection in the etiology of atherosclerosis--the case for Chlamydia pneumoniae. Clin Cardiol. 1997 Oct; 20(10):829-36.

41. Martin D, Bursill J, Qui MR, Breit SN, Campbell T. Alternative hypothesis for efficacy of macrolides in acute coronary syndromes. Lancet. 1998 Jun 20;351 (9119):1858-1859.

42. Sinisalo J, Mattila K, Valtonen V, Anttonen O, Juvonen $\mathrm{J}$, Melin J, et al. Effect of 3 months of antimicrobial treatment with clarithromycin in acute non-q-wave coronary syndrome. Circulation 2002;(105):1555-60.

43. Scheller B, Hennen B, Markwirth T, Schieffer H. Evaluation of the role of Chlamydia pneumoniae in the pathogenesis of atherosclerosis - a review. J Clin Basic Cardiol 2000; 3: 155.

44. Grayston JT, Kronmal RA, Jackson LA, Parisi AF, Muhlestein JB, Cohen JD, et al. Azithromycin for the secondary prevention of coronary events. N Engl J Med. 2005 Apr 21; 352(16):1637-45.

45. Anderson JL, Muhlestein JB, Carlquist J, Allen A, Trehan $\mathrm{S}$, Nielson $\mathrm{C}$, et al. Randomized secondary prevention trial of azithromycin in patients with coronary artery disease and serological evidence for Chlamydia pneumoniae infection: The Azithromycin in Coronary Artery Disease: Elimination of Myocardial Infection with Chlamydia (ACADEMIC) study. Circulation. 1999 Mar 30; 99(12):1540-7.

46. Cercek B, Shah PK, Noc M, Zahger D, Zeymer U, Matetzky S, et al. Effect of short-term treatment with azithromycin on recurrent ischaemic events in patients with acute coronary syndrome in the Azithromycin in Acute Coronary Syndrome (AZACS) trial: a randomised controlled trial. Lancet 2003; (361):809-13.

47. O'Connor CM, Dunne MW, Pfeffer MA, Muhlestein JB, Yao L, Gupta S, et al. Azithromycin for the secondary prevention of coronary heart disease events: the WIZARD Study: a randomized controlled trial. JAMA 2003; (290):1459-66.

48. Zahn R, Schneider S, Frilling B, et al. Antibiotic therapy after acute myocardial infarction: A prospective randomized study. Circulation. 2003;107: 1253-1259.

49. Leowattana W, Bhuripanyo K, Singhaviranon L, et al. Roxithromycin in prevention of acute coronary syndrome associated with Chlamydia pneumoniae infection: a randomized placebo controlled trial. J Med Assoc Thai. 2001; 84 (S3): S669-S675.

50. Sander D, Winbeck K, Klingelhöfer J, Etgen T, Conrad B. Progression of early carotid atherosclerosis is only temporarily reduced after antibiotic treatment of Chlamydia pneumoniae seropositivity. Circulation. 2004 Mar;109(8):1010-5.

51. Mosorin M, Juvonen J, Biancari F, et al. Use of doxycycline to decrease the growth rate of abdominal aortic aneurysms: a randomized, double-blind, placebocontrolled pilot study. J Vasc Surg. 2001; 34: 606-610.

52. Vammen S, Lindholt JS, Ostergaard L, Fasting H, Henneberg EW. Randomized double-blind controlled trial of roxithromycin for prevention of abdominal aortic aneurysm expansion. Br J Surg. 2001 Aug;88(8):1066-72.

53. Berg HF, Maraha B, Scheffer GJ, Quarles-van Ufford M, Vandenbroucke-Grauls CM, Peeters MF Treatment with clarithromycin prior to coronary artery bypass graft surgery does not prevent subsequent cardiac events. Clin Infect Dis. 2005 Feb 1;40(3):358-65. 
54. Stone AF, Mendall MA, Kaski JC, et al. Effect of treatment for Chlamydia pneumoniae and Helicobacter pylori on markers of inflammation and cardiac events in patients with acute coronary syndromes: South Thames Trial of Antibiotics in Myocardial Infarction and Unstable Angina (STAMINA).Circulation. 2002; 106: 1219-1223.
55. Libby P, Egan D, Skarlatos S. Roles of infectious agents in atherosclerosis and restenosis: an assessment of the evidence and need for future research. Circulation. 1997 Dec 2;96 (11):4095-103.

Para citar este artículo: Gudiño-Gomezjurado A, Gudiño-Gomezjurado M. Ateroesclerosis asociada a infección por Chlamydophila pneumoniae: interacción entre el ser humano y una bacteria. Duazary. 2015 jun; 12 (1): 41 - 48 